\title{
Evoked and induced oscillatory activity contributes to abnormal auditory sensory gating in schizophrenia
}

\author{
Tzvetan Popov ${ }^{\mathrm{a}, *}$, Todor Jordanov ${ }^{\mathrm{a}}$, Nathan Weisz ${ }^{\mathrm{a}}$, Thomas Elbert ${ }^{\mathrm{a}}$, \\ Brigitte Rockstroh ${ }^{\mathrm{a}}$, Gregory A. Miller ${ }^{\mathrm{a}, \mathrm{b}}$ \\ a Department of Psychology, University of Konstanz, Germany \\ ${ }^{\circ}$ Departments of Psychology and Psychiatry and Beckman Institute for Biomedical Imaging, University of Illinois at Urbana-Champaign, Urbana, II, USA
}

\begin{abstract}
A B S T R A C T
The ratio of magnetoencephalogram-recorded brain responses occurring $50 \mathrm{~ms}$ after paired clicks (S2-evoked M50/S1-evoked M50) serves as a measure of sensory gating. An abnormally large ratio is commonly found in schizophrenia. Whether this abnormality indicates impaired gating is debated. Using event-related oscillations the present study sought to elucidate processes contributing to the phenomenon of altered M50 gating ratio. Schizophrenia inpatients $(n=50)$ showed the expected large M50 gating ratio relative to 48 healthy controls, which correlated with less induced frontally generated activity in the 10-15 Hz frequency band starting $200 \mathrm{~ms}$ before the onset of S2. Patients also produced smaller alpha $(8-12 \mathrm{~Hz})$ and gamma $(60-80 \mathrm{~Hz})$ responses to S1. Results suggest that the deviant gating ratio in schizophrenia is a consequence of a complex alteration in the processing of incoming information that cannot be attributed to impaired gating alone.
\end{abstract}

Schizophrenia

Magnetoencephalography

Sensory gating

Oscillatory rhythm

Event-related desynchronization

\section{Introduction}

Sensory gating refers to a phenomenon of cortical response suppression to the second of two identical stimuli presented in rapid succession. This response attenuation is thought to reflect the ability of the brain to inhibit the processing of repeated information, a mechanism that protects the processing of irrelevant information (Adler et al., 1982; Boutros et al., 2009; Clementz et al., 1997; Edgar et al., 2008; Freedman et al., 1987; Huang et al., 2003). In the auditory modality, sensory gating has usually been studied in a paired-click paradigm: two brief click stimuli are presented with 500 ms stimulus onset asynchrony. Both stimuli elicit a response at around $50 \mathrm{~ms}$ (P50 electroencephalographic event-related potential or M50 magnetoencephalographic evoked field), with the response to the second stimulus attenuated in comparison to the first one. The amplitude ratio (S2-evoked P50/S1-evoked P50) commonly serves as a measure of sensory gating.

Schizophrenia samples consistently show a higher ratio than the comparison samples, which is interpreted as a sign of deficient or inefficient sensory gating (Adler et al., 1982; Patterson et al., 2008; Yee et al., 2010). The inability to suppress distracting, irrelevant

* Corresponding author at: Department of Psychology, University of Konstanz, PO Box D23, D-78457 Konstanz, Germany. Fax: +497531 884601 E-mail address: Tzvetan.Popov@uni-konstanz.de (T. Popov). information is proposed to contribute to the symptoms of schizophrenia (Boutros et al., 2009; Bramon et al., 2004). Associations with measures of neuropsychological impairment (Potter et al., 2006; Thoma et al., 2006; Thoma et al., 2003) support the interpretation of less efficient sensory gating in schizophrenia. Yet the reason for an exaggerated ratio in schizophrenia remains unclear, and the mechanisms leading to the suppression of the response to $\mathrm{S} 2$ are not yet identified. The $\mathbf{S} 2 / \mathrm{S} 1$ ratio is potentially sensitive to the encoding processes related to $\mathrm{S} 1$ as well as the inhibition processes related to S2, with the relative contributions currently controversial (Clementz and Blumenfeld, 2001; Edgar et al., 2008; Hall et al., 2010; Hong et al., 2004), In particular, it is unclear whether the abnormal gating ratios in schizophrenia reflect dysfunctional encoding, dysfunctional gating, or both.

Oscillatory phenomena in response to $\mathrm{S} 1$ and to $\mathrm{S} 2$ provide additional information about stimulus encoding (Tallon-Baudry et al., 1999) and active memory (Pulvermuller et al., 1999) and thus may be useful to specify the abnormal M50 gating ratio in schizophrenia. As suggested by Jensen and Mazaheri (2010), the power increase in the gamma frequency range $(30-100 \mathrm{~Hz})$ reflects task engagement and active processing and is typically accompanied by a power decrease in the alpha frequency range $(8-12 \mathrm{~Hz})$, the latter also known as eventrelated desynchronization (ERD). Moreover, Krause (2006) showed that the encoding of acoustic material elicited alpha power increase, whereas the recognition and retrieval of the same stimulus material was associated with alpha ERD. Indeed, abnormalities have been 
reported for several frequency bands and have been related to sensory and cognitive functions (Haenschel et al., 2009; Kissler et al., 2000 White et al., 2010). In the paired-stimulus gating design, schizophrenia patients usually display reduced activity in lower frequency bands whereas alterations in higher frequency bands are less frequently reported (Brenner et al., 2009; Brockhaus-Dumke et al., 2008; Clementz and Blumenfeld, 2001; Edgar et al., 2008; Hall et al., 2010; Hong et al., 2004, 2008; Rosburg et al., 2009). These results suggest that a more detailed mapping of the oscillatory activity might help to determine the mechanisms contributing to the P50/M50 gating ratio and its alteration in schizophrenia. In particular, the induced oscillatory activity has received little attention in the literature on the sensory gating deficit in schizophrenia.

The present study used high-density magnetoencephalography (MEG) to examine evoked and induced oscillatory activity in a pairedclick design in schizophrenia patients and healthy controls. In particular, we hypothesize that deficient stimulus encoding in schizophrenia should be manifest in the weaker evoked gamma activity in patients than in healthy control subjects and that deficient recognition and retrieval of the encoding of $\mathrm{S} 1$ in the preparation of S2 processing should be manifest in the weaker induced alpha power decrease in schizophrenia patients than in controls. We further hypothesize that these group differences in oscillatory activity should influence the relationship between evoked and induced activity and the M50 gating ratio: reduced evoked gamma power increase and reduced induced alpha power decrease should vary with the abnormal M50 ratio. Moreover, we hypothesized that the cortical sources of evoked and induced oscillatory activity extending beyond those of the M50 generator structures (in particular to the frontocortical regions) indicate the influences of oscillatory activity involved in the fronto-cortical top-down modulation of event-related potentials. The support for these hypotheses should explain the abnormal $\mathrm{M} 50$ ratio as a consequence of insufficiently processed S2 and thereby help to understand the altered sensory gating in schizophrenia.

\section{Methods}

\section{Participants}

The study was approved by the Ethics Committee of the University of Konstanz. The sample included 50 stable inpatients recruited, evaluated, and treated at the local centre for psychiatry (age $M=30.2$, $\mathrm{SD}=7.9,5$ female) and 48 healthy control subjects (age $M=28.1$, $\mathrm{SD}=6.2,15$ female). The inclusion criteria for patients were ICD-10 diagnoses of schizophrenia,

function, and no history of neurological disorders or head trauma with loss of consciousness. The control participants were included if they did not meet the criteria for a lifetime diagnosis of mental illness (screened with the MINI interview; Ackenheil et al., 1999), did not report any history of head trauma with loss of consciousness, and were free of psychoactive medication. Right-handedness was confirmed for 41 patients and 44 controls according to the Edinburgh Handedness Inventory (Oldfield, 1971), five patients and three controls were left-handed, and four patients and one control were ambidextrous. All participants gave written informed consent for the participation following a complete description of the study protocol and measurements. All participants refrained from smoking for at least one hour prior to MEG recording. Participants received $20 €$ for participation in the experiment, which lasted for about $2 \mathrm{~h}$.

${ }^{1}$ Subcategories paranoid-hallucinatory schizophrenia (F20.0, $n=40$ ), disorganized schizophrenia $(F 20.1, n=2)$, post-schizophrenic depression $(F 20.4, n=2)$, residual schizophrenia (F20.5, $n=1$ ), acute polymorphic psychotic disorder with symptoms of schizophrenia (F23.1, $n=3$ ), and schizoaffective disorder (F25.1, n=2). Results of an intervention trial on a subset of this sample are reported in Popov et al. (2011).

\section{Design and data acquisition}

One hundred pairs of $3 \mathrm{~ms}$ square-wave clicks were presented with a $500 \mathrm{~ms}$ onset-to-onset inter-stimulus interval and an $8 \mathrm{~s}$ jittered inter-trial interval (offset to onset 7-9 s). Clicks were presented at $50 \mathrm{~dB}$ above subjective hearing level, determined separately for each ear, and delivered via non-ferromagnetic tubes of about $5 \mathrm{~m}$ length. No task was involved, except that participants were asked to keep their eyes focused on a small fixation point throughout the measurement.

MEG was recorded while subjects were in a supine position, using a 148-channel magnetometer (MAGNES ${ }^{\mathrm{TM}} 2500 \mathrm{WH}, 4 \mathrm{D}$ Neuroimaging, San Diego, USA). Data were continuously recorded with a sampling rate of $678.17 \mathrm{~Hz}$ and a bandpass filter of 0.1 to $200 \mathrm{~Hz}$. For artifact control, the vertical and horizontal electrooculogram (EOG recorded from four electrodes placed near the left and right temporal canthus and above and below the right eye) were recorded using a SynAmps amplifier (NEUROSCAN Laboratories, Sterling, VA, USA). The subject's nasion, left and right ear canal, and head shape were digitized with a Polhemus 3 Space $\circledast$ Fasttrack prior to each session.

\section{Data reduction and analysis}

Global noise was removed offline from the MEG data by subtracting external, non-biological noise recorded by 11 MEG reference channels. Before the subtraction, reference channels were multiplied with individually calculated fixed weight factors.

\section{Spectral analysis}

Epochs of 1000 ms before and 2000 ms following S1 were identified from the continuous recordings, and the eye blink and muscle artifacts contaminated trials were rejected. Patients $(M=84.1, S D=10.7)$ and controls $(M=83.4, S D=11.4)$ did not differ in the number of trials. Spectral analysis was performed according to the procedures described by Tallon-Baudry et al. (1997) and implemented in Fieldtrip, an opensource MEG/EEG signal processing toolbox for Matlab (http://www. fieldtrip.fcdonders.nl/). Convolution with a complex Morlet wavelet was applied to single trials: $w\left(t, f_{0}\right)=A \exp \left(-t^{2} / 2 \sigma_{t}^{2}\right) \exp \left(2 i \pi f_{0} t\right)$, where $\sigma_{t}=m / 2 \pi f_{0}, i$ was the imaginary unit, and $A=\left(\sigma_{t} \sqrt{ } \pi\right)^{-1 / 2}$ was the normalization factor. The trade-off between frequency and time resolution was determined by the constant $m=7$. The time-frequency representation of power (TFR) was calculated by averaging the squared absolute values of the convolutions over trials. The TFR of post-stimulus activity (pst $A$ ) was expressed as the change relative to the pre-stimulus activity (preA): (pstA-preA)/preA. This procedure yields time-frequency representations containing evoked as well as induced responses.

Relevant time-frequency windows were defined using a clusterbased, independent-sample t-test with Monte Carlo randomization following the procedure described in Maris and Oostenveld (2007). This procedure effectively controls for multiple comparisons and allows the identification of sensor clusters with significant group differences in 3D on a sensor level (time, frequency, and sensors) and a brain source level (volume). At least 3 sensors/cluster were required from 1000 randomizations for the time-frequency data, and 500 randomizations were required for the comparisons of voxel-clusters at the source level. Sensor/voxel clusters were identified as differentially active when group differences exceeded a threshold of significance at the $5 \%$ level; the test statistic was defined as the sum of the t-statistics of the sensors/voxels within the respective cluster.

A frequency-domain adaptive spatial filtering algorithm enabling the dynamic imaging of the coherent sources (DICS; Gross et al., 2001) served to estimate the sources of activity that contributed to the effects at the sensor level. This algorithm uses cross-spectral density matrices obtained from the data to construct a spatial filter optimized for a specific location (voxel). The time windows and frequency bands of interest were based on the results obtained for the sensor clusters. 
A realistic single-shell brain model (Nolte, 2003) was constructed for each subject based either on the individual structural magnetic resonance (MR) images (available for 36 controls and 17 schizophrenia patients) or on an affine transformation of an MNI-template brain (Montreal Neurological Institute (MNI), Montreal, Canada; http:// www.bic.mni.mcgill.ca/brainweb) to the subject's digitized individual head shape (for 12 controls and 33 patients; see also Keil et al., 2010; Lecaignard et al., 2008). The results for individual subjects were normalized onto a common brain template for illustration (e.g., source grand averaging) and for the statistical group comparisons.

\section{Event-related analysis}

Epochs used for the averaging of the scalp-sensor data across trials were defined as $300 \mathrm{~ms}$ pre-S1 baseline and $1000 \mathrm{~ms}$ following S1, thus $500 \mathrm{~ms}$ following S2. Epochs with amplitude $>3500 \mathrm{fT}$ and/ or gradients $>2500 \mathrm{fr} /$ sample were rejected. Patients $(M=86.2$, $S D=13.3)$ and controls $(M=90.3, S D=9.7)$ did not differ in the number of trials accepted. Artifact-free epochs were averaged and filtered with a 1-45 $\mathrm{Hz}$ bandpass filter (high pass: $12 \mathrm{~dB} /$ octave, zero phase; low pass: $24 \mathrm{~dB}$ /octave, zero phase). M50 was defined as the segment of the event-related field (ERF) within a time window 40$80 \mathrm{~ms}$ after the stimulus onset with the largest amplitude prior to M100. The visual inspection of the ERF ensured auditory cortical activation, dipolar topographic distribution with ingoing and outgoing magnetic fields, and corresponding polarity reversal and topographic distribution opposite in direction to that of M100. Based on these sensor data, sources were estimated by fitting two regional dipoles simultaneously in the left and right hemispheres for a 20 -ms interval around the M50 peak. For calculation of the auditory gating ratio, the strength of the dipole source after S2 was divided by the strength after S1. Event-related analysis was performed using BESA 5.2 (http://www.besa.de).
Correlation analysis

The relationships between spectral characteristics and M50 gating ratio were probed via Pearson correlations: for each individual the gating ratio was correlated with a set of power spectra that included every time-frequency bin for every MEG sensor. The resulting distributions of significant coefficients for distinct time-frequency bins were plotted (a) as a function of time and (b) as a function of sensor cluster, i.e., scalp topography. The significant time-frequency windows showing a relationship with the auditory gating ratios on sensor level were then submitted to a source reconstruction. The estimated activity relative to the baseline period resulting in a $3 \times \mathrm{N}$ volume matrix per subject was correlated with the individual M50 gating ratio, where significant clusters of voxels with the correlation coefficients between relative power and gating ratio were determined after the Monte Carlo randomization at the $5 \%$ level.

\section{Results}

Stimulus-evoked and induced oscillatory activity

As illustrated in Fig. 1A, S1 and S2 elicited the largest changes from the baseline in lower frequencies $(<30 \mathrm{~Hz})$. Power increased in response to $\mathrm{S} 1$ onset in both groups, this event-related synchronization (ERS) reaching its maximum around $100 \mathrm{~ms}$ post S1 onset. Additional sources of evoked alpha activity in the primary auditory cortices did not differ by group (Fig. 1B, top two rows). The time course of power in the alpha $(8-12 \mathrm{~Hz})$ frequency band averaged across the frontotemporal and posterior sensors, illustrated in Fig. 2A, indicates that the S1-related ERS was more pronounced in controls than in schizophrenia patients. The nonparametric permutation test verified a group difference for this frequency band $50-200 \mathrm{~ms}$ after $\mathrm{S} 1$ at the right fronto-temporal sensors ( $p=0.03$; Fig. $2 B$ ). DICS for this
A
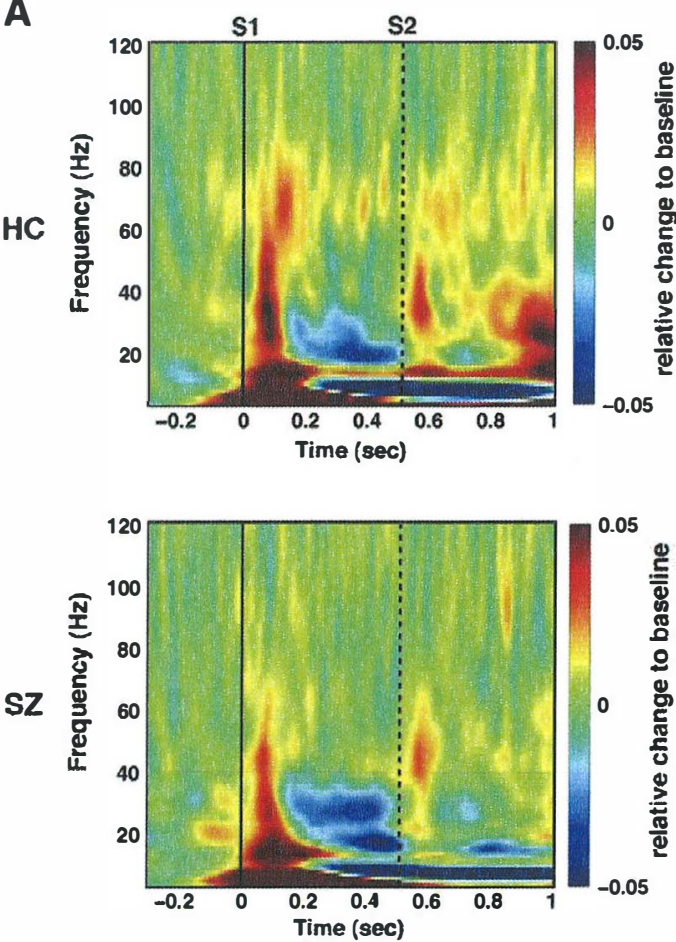

B
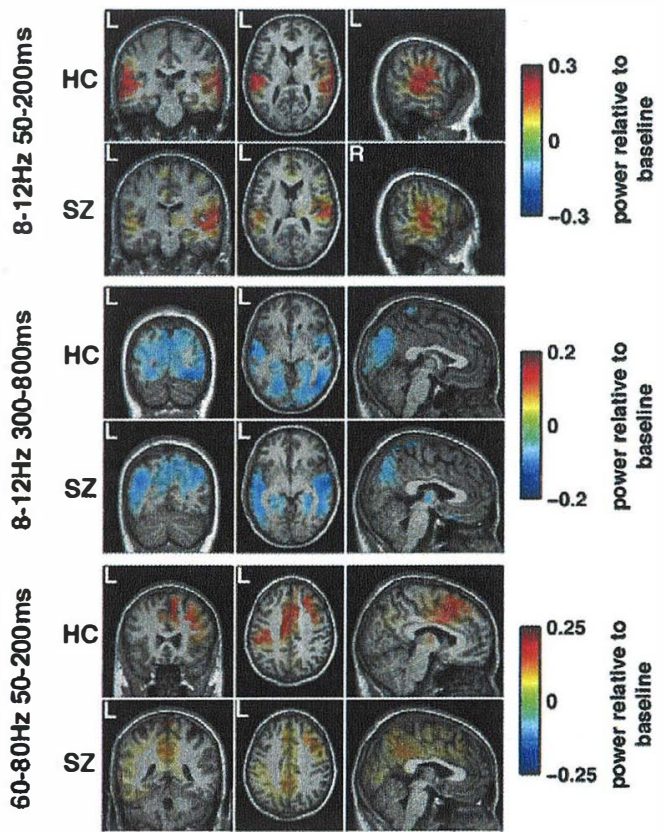

Fig. 1. A: time-frequency representation of relative power changes averaged over all sensors for the healthy control group (HC, top) and the schizophrenia group (SZ, bottom). B: source distribution of averaged oscillatory activity ref erred to baseline period for 8-12 Hz activity 50-200 ms after S1 onset (top), 8-12 Hz activity $300-500$ ms after S1 (middle), and $60-80 \mathrm{~Hz}$ activity $50-200 \mathrm{~ms}$ after $\mathrm{S} 1$ onset (bottom). Source distributions are displayed separately for healthy controls (HC) and schizophrenia patients (SZ). Color bars indicate changes in power from baseline, green color indicating no change, red color indicating more power, and blue color indicating less power. 


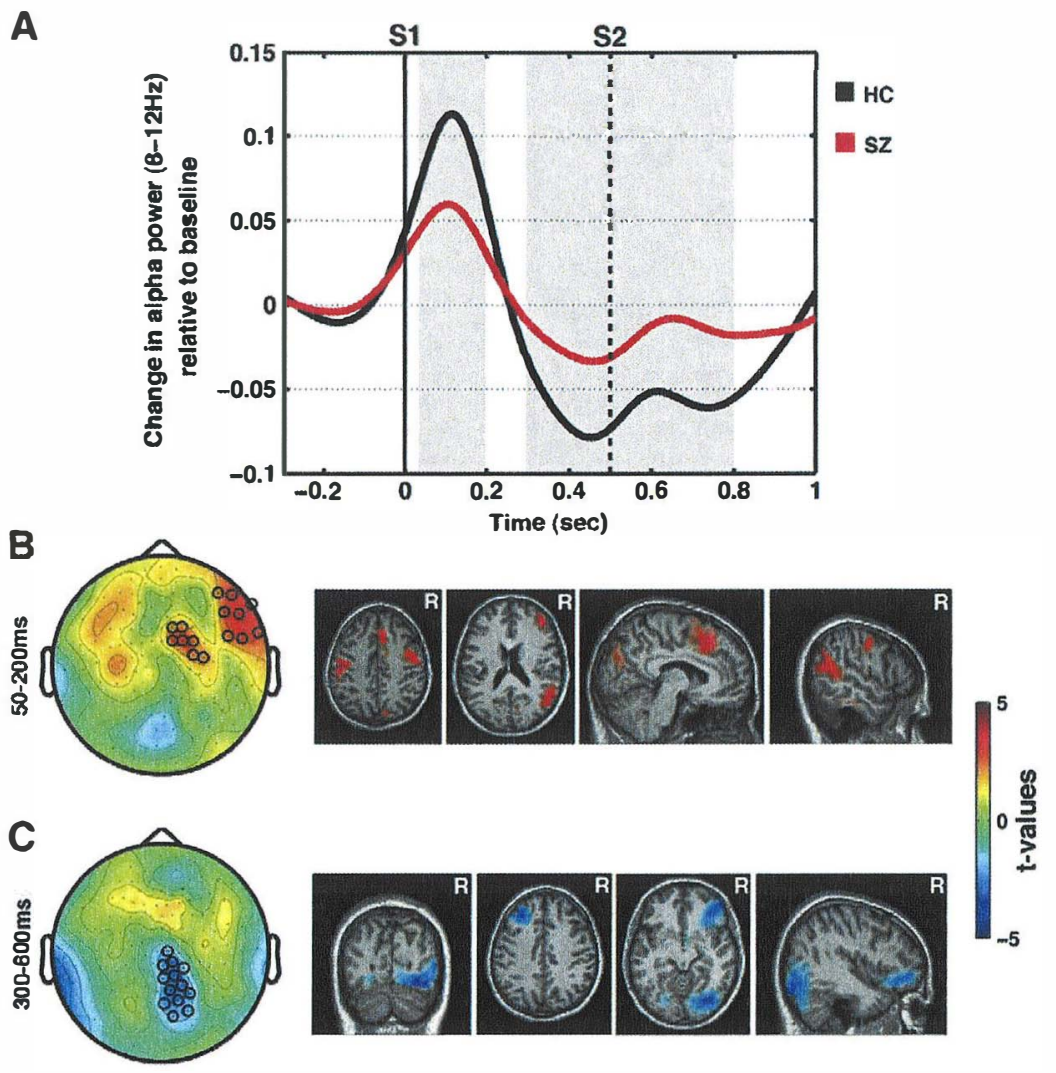

Fig. 2. A: time course of event-related synchronization (ERS; increased power) in the 8-12 Hz band and event-related desynchronization (ERD; decreased power) in the 8-12 $\mathrm{Hz}$ band, averaged across fronto-temporal and posterior sensors separately for healthy controls (black line) and schizophrenia patients (red line). B (left): Topographical distribution of significant sensor clusters $50-200 \mathrm{~ms}$ (B left, $\mathrm{p}=0.03$ ) and $300-800 \mathrm{~ms}$ ( $\mathrm{Cleft}, \mathrm{p}=0.03$ ) after $\mathrm{S} 1$ onset. The color bar indicates statistical effects by a range of $\mathrm{t}-\mathrm{values}$, red colorsignaling positive $\mathrm{t}-\mathrm{values}$. $\mathrm{B}$ and $\mathrm{C}$, right: statistical map of differences between groups, with differences being computed on the relative change of power to baseline as suggested by DICS forthe time windows $50-200$ ms (B) and $300-800 \mathrm{~ms}(C)$.

time-frequency window (Fig. 2B) indicated that the group differences originated from the right and left fronto-temporal regions, right midtemporal gyrus, and the middle part of the cingulum.

Alpha-band $(8-12 \mathrm{~Hz})$ power decreased in the interval of 300 $800 \mathrm{~ms}$. This event-related desynchronization (ERD), which reached its maximum shortly before or at S2 onset (Fig. 2A), was more pronounced in controls than in patients. The nonparametric permutation test verified that this decrease was particularly pronounced at posterior sensors ( $p=0.03$; Fig. $2 C)$. Descriptively, it is apparent in Fig. $2 \mathrm{~A}$ that the alpha activity increased again approximately $100 \mathrm{~ms}$ following the $\mathrm{S} 2$ onset. This suggests that responses to $\mathrm{S} 2$ were evoked on top of the ongoing induced alpha desynchronization that was triggered by S1. The source reconstruction (Fig. $2 \mathrm{C}$ ) suggested right occipito-temporal regions (including lingual gyrus, fusiform gyrus and inferior occipital gyrus) and left and right prefrontal regions (left middle frontal gyrus and right orbitofrontal cortex) contributing to the group difference in ERD. As already mentioned for ERS, the sources of induced $8-12 \mathrm{~Hz}$ activity identified in the primary auditory cortices and in distributed posterior brain areas did not differentiate the groups (Fig. 1B).

$\mathrm{S} 1$ and S2 also evoked changes at frequencies $>30 \mathrm{~Hz}$. The evoked $60-80 \mathrm{~Hz}$ gamma-band response was prominent in controls 50 $200 \mathrm{~ms}$ after S1, whereas patients showed almost no gamma-band change (Fig. 3A). The nonparametric permutation test confirmed this effect over the fronto-central sensors (Fig. 3B, left; $p=0.002$ ). DICS (Fig. 3B, right) indicates that the group effect resulted from the oscillatory activity in the left frontocentral brain regions including the left superior frontal gyrus, left middle frontal gyrus, and left precentral sulcus.
A
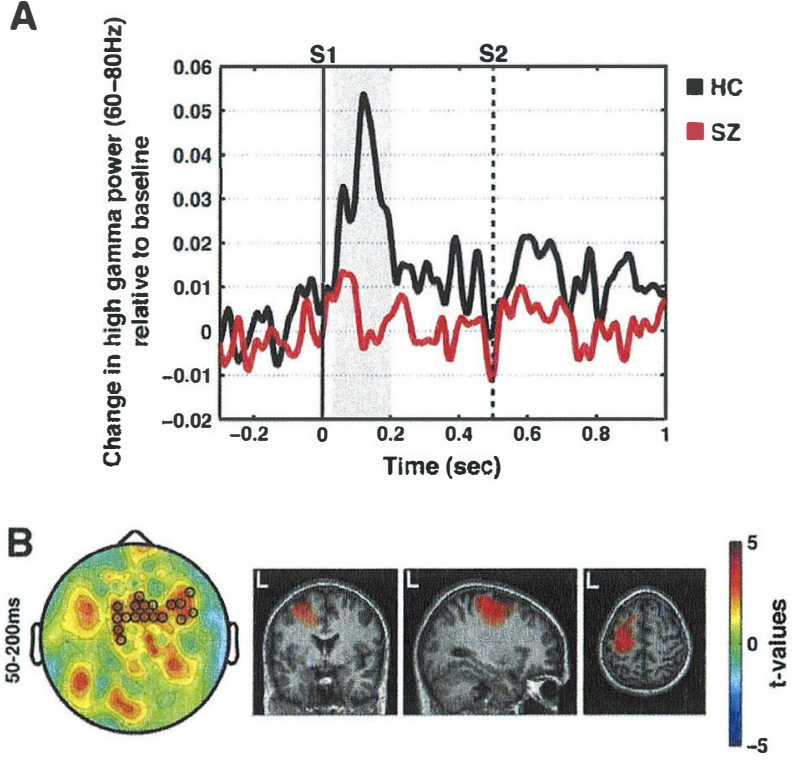

Fig. 3. A: Time course of evoked $60-80 \mathrm{~Hz}$ gamma response to $\mathrm{S} 1$ averaged across frontocentral sensors separately for healthy controls $(\mathrm{HC})$ and schizophrenia patients (SZ). B left: topographical distribution of the significant sensor cluster $(p=0.002) 50$ $200 \mathrm{~ms}$ after S1 onset. B right: statistical map of differences between groups, with differences being computed on the relative change of power to baseline as suggested by DICS. The color bar indicates the range of t-values. 


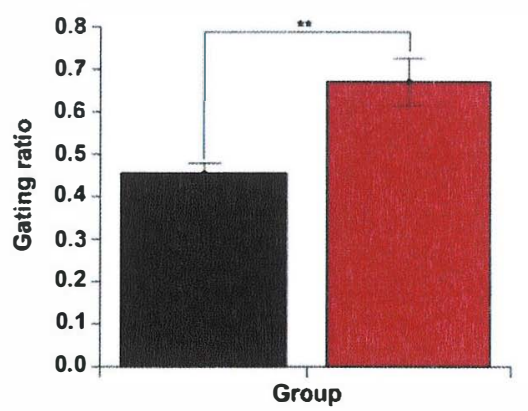

HSC

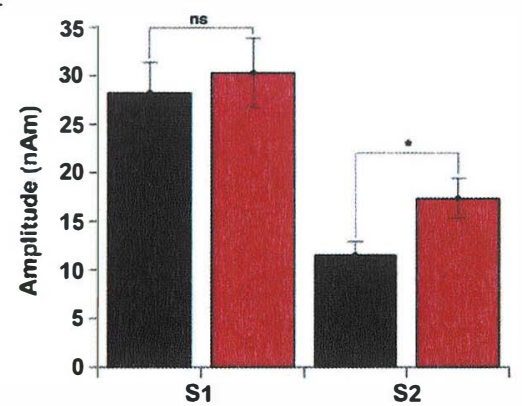

Fig. 4. Mean auditory dipole sensory gating ratios (left) and mean amplitudes of M50 dipole sources after S1 and S2 onset (right) for healthy controls (black bars) and schizophrenia patients (red bars). Error bars indicate $1 \mathrm{SE}$; significant group differences are marked by asterisks: ${ }^{* *} \mathrm{p}<0.01 ;{ }^{*} \mathrm{p}<0.05$.

Relationship between M50 gating ratio and evoked and induced oscillatory activity

A Group $\times$ Hemisphere analysis of variance indicated higher gating ratio for patients than for controls $(F(1,96)=12.29$, $\mathrm{p}<.001$; Fig. 4, left). To explore this finding, the comparisons of separate S1 and S2 scores confirmed a larger S2 response in patients than in controls $(\mathrm{F}(1,96)=$ 5.35, $\mathrm{p}<.03$, Fig. 4, right) but no difference at $\mathrm{S} 1(\mathrm{~F}<1)$. There were no Hemisphere or Group $\times$ Hemisphere gating-ratio effects. Therefore, gating ratios were averaged over the hemispheres for subsequent analysis.

Correlation analyses (see Correlation analysis Section) demonstrated the relationships between gating ratio and induced oscillatory activity in the $10-15 \mathrm{~Hz}$ band in patients but not in controls but relationships between gating ratio and $60-80 \mathrm{~Hz}$ evoked activity in controls but not in patients. In patients (Fig. 5), higher M50 gating ratio varied with the lower induced $10-15 \mathrm{~Hz}$ desynchronization prior to S2 onset (see Correlation analysis Sectionstep (a) and rectangle in Fig. 5A). Evident in the topographical distribution of correlation coefficients (Correlation analysis Section step (b) and Fig. 5B), relationships were significant at posterior and fronto-temporal sensors $(p=0.004)$. This relationship was also confirmed in source analyses (Correlation analysis Section step (c) and Fig. 5C) suggesting generators contributing to these correlations in brain regions including cuneus, pre-cuneus, left occipital inferior gyrus, left lingual gyrus, and medial prefrontal cortex.

In controls (Fig. 6), higher S1-evoked gamma power was related to lower gating ratios (rectangle in Fig. 6A). The plotting correlation coefficient as a function of topography emphasized significant relationships at the sensor clusters over the left centro-parietal and right frontotemporal regions (Fig. 6B). DIC S confirmed this relationship for sources
A

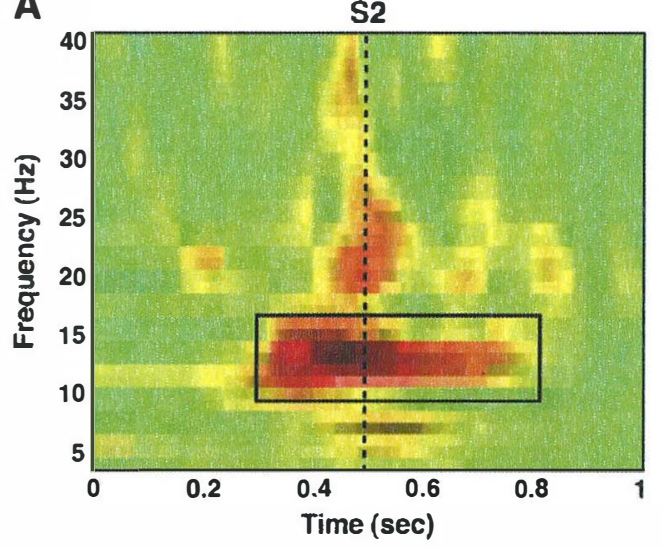

B

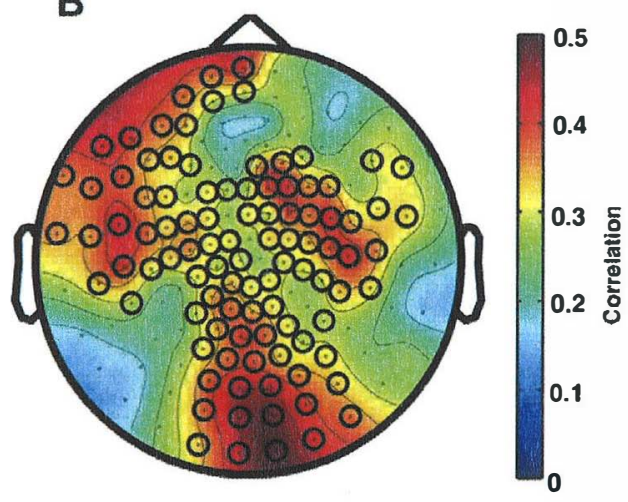

C
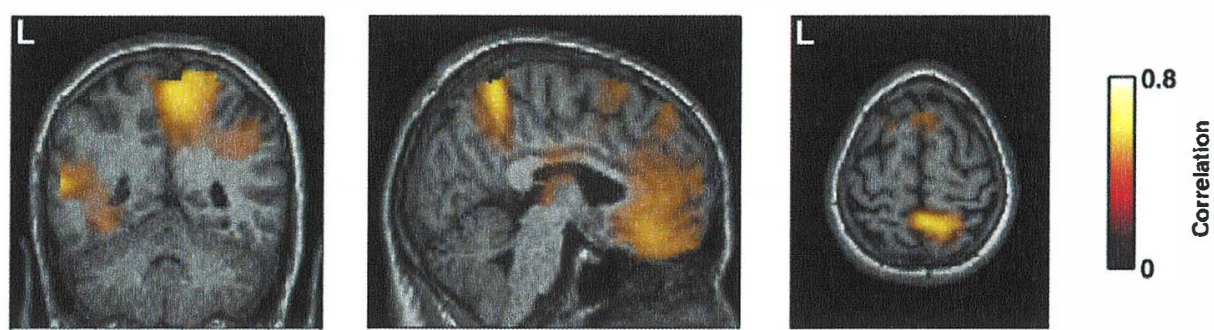

Fig. 5. A: correlations between relative power in each time-f requency analysis bin and M50 ratios (dipole strength of S2-evoked M50 divided by the dipole strength of S1-evoked M50) in schizophrenia patients, plotted for the significant sensor cluster in panel B. B: correlations between power in the 10-15 $\mathrm{Hz}$ band in the $300-800 \mathrm{~ms}$ time window and M50 gating ratios in schizophrenia patients are plotted as a function of topography for the time-frequency window emphasized in panel A. Open black circles mark individual sensors belonging to a significant cluster, red colored areas mark distribution of significant correlation coefficients. C: distribution of correlation coefficients within significant voxel clusters (corrected $\mathrm{p}<0.05)$ between the relative change of power to the baseline $(10-15 \mathrm{~Hz}, 300-800 \mathrm{~ms})$ and M50 ratios. 
A

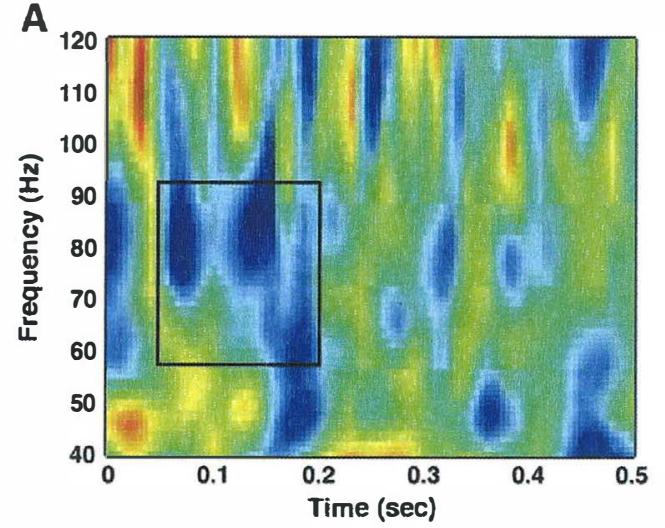

B

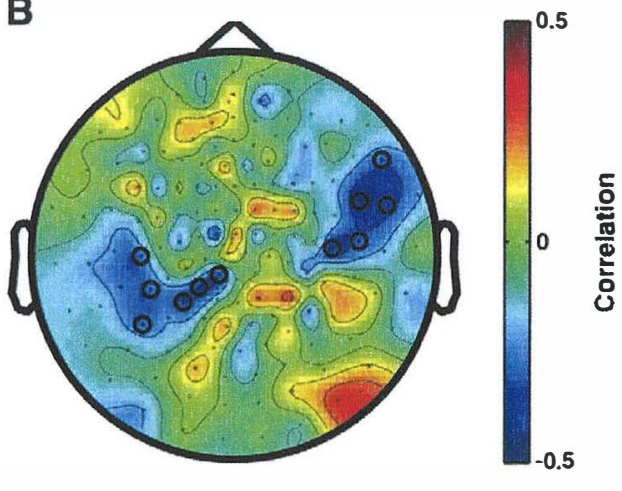

C
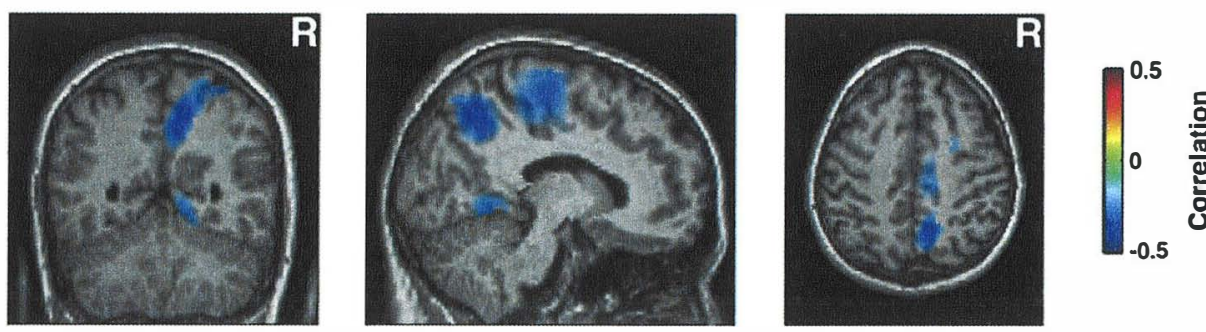

Fig. 6. A: correlations between the relative power and M50 ratios (dipole strength of S2-evoked M50 divided by the dipole strength of S1-evoked M50) in the control subjects, plotted for the significant sensor cluster in panel B. B: correlations between power in the $60-80 \mathrm{~Hz}$ band in the 50-200 ms time window and M50 gating ratios in the control subjects are plotted as a function of topography for the highlighted time-f requency window in panel A. Open black circles mark individual sensors belonging to a significant cluster, blue colored areas mark distribution of significant correlation coefficients. C: distribution of correlation coefficients within significant voxel clusters ( $p<0.05$, corrected) between the relative change of power to the baseline $(60-80 \mathrm{~Hz}, 50-200 \mathrm{~ms})$ and the M50 gating ratios.

primarily in the parietal brain regions, i.e. cuneus and posterior cingulate cortex (Fig. 6C).

\section{Discussion}

Evoked and induced oscillatory activity was examined in a paired stimulus design with the aim to elucidate processes contributing to altered M50 gating ratios in schizophrenia patients. The present results replicated the oft-reported gating deficit in schizophrenia, here assessed in the source space. Importantly, patients and controls differed in the stimulus-locked (evoked) changes in the alpha and gamma bands and non-phase-locked (induced) changes in alpha activity during the inter-stimulus interval. Patients' reduced evoked and induced oscillatory activity was related to their higher M50 gating ratio. The concept of sensory gating cannot fully explain this outcome. Hence, several other mechanisms of auditory processing must be involved in producing these abnormalities in schizophrenia.

The present findings of smaller evoked alpha- and upper gammaband responses to the first auditory stimulus may indicate less efficient encoding or less efficient active memory for this stimulus. White et al. (2010) reported altered alpha and gamma activity to be related to the impaired somatosensory processing in schizophrenia. Thus, oscillatory activities in these frequency bands may indicate stimulus encoding regardless of stimulus modality. Generator sources of the evoked gamma response were found in the prefrontal brain regions (middle frontal gyrus and dorsal ACC), suggesting top-down modulation of initial auditory information processing. Similar reduced power and phase-locking of the early evoked gamma responses to auditory stimuli in schizophrenia have been reported by Leicht et al. (2010). Whereas Leicht and colleagues attributed the reduced gamma response to reduced activity in the medial frontal gyrus and ACC, Mulert et al. (2007) related the gamma band activity in dorsal ACC to the top-down influences on early auditory processing. The markedly reduced evoked gamma response in schizophrenia patients suggests that this modulation was impaired.

A second contributor to sensory gating and its disruption in schizophrenia may be that the induced alpha desynchronization before the onset of S2 is a consequence of S1-initiated attention binding. Auditory gating ratios can be modified by the attention modulation in schizophrenia patients, supporting the hypothesis of insufficient recruitment of automatic $\mathrm{S} 1$-initiated attention in patients (Yee et al., 2010). Although the present results did not confirm smaller S1-evoked M50 in patients, the reduced S1-evoked alpha and gamma responses may reflect less activation of the attention resources (Debener et al., 2003; Tallon-Baudry et al., 2005), with less induced alpha desynchronization being a consequence.

Although the present paired-click design did not include an explicit performance task, S1 must have initiated memory trace formation and working memory processes (Lijffijt et al., 2009), thereby modulating alpha desynchronization. Event-related or oscillatory manifestation of stimulus recognition and comparison with the memory trace may be expected following (rather than before) S2onset. Nevertheless, alpha desynchronization preceding S2 onset may reflect a preparatory state facilitating the comparison and memory retrieval of S2. Accordingly, a third candidate mechanism is that less desynchronization in patients may reflect less efficient working memory processes. Association with memory-related processes is also supported by the frontocortical generators that contributed to group differences in alpha desynchronization. Moreover, induced gamma responses have been associated with active memory processes (Pulvermuller et al., 1999), so the smaller evoked gamma response in patients may have reflected less efficient active memory processes.

The source reconstruction revealed group differences in evoked and induced oscillatory activity in distributed generator structures, including the frontocortical and posterior sources, even though the 
normal sources of M50 and evoked alpha activity were found in the auditory cortices. The frontocortical sources during the processing of auditory stimuli have been implicated in the MEG studies of nonpatients (Mayer et al., 2009; Weisser et al., 2001) and intracranial recordings in epileptic patients (Grunwald et al., 2003; Korzyukov et al., 2007). Moreover, Williams et al. (2011) reported EEG evidence of an association between enhanced gating-related dipole activity in the dorsolateral prefrontal cortex and higher gating ratios in schizophrenia subjects, which might point to a relationship between working memory and sensory gating.

In concert, the interplay of oscillatory findings suggests that the gating ratio varies as a function of the engagement of distributed cortical networks in initial stimulus encoding, sustained attention, and elaborative processing. Some evidence suggests that the fidelity of the neural representations is reduced in schizophrenia (e.g., Popov et al., 2011). Consequently, evoked activity, which in effect is the resonance of this representation to the incoming stimulus input, is smaller. Hence, the indices of active memory and with it the gamma response are smaller when the fidelity is lower. The second stimulus would normally reactivate the same cell assemblies. Presented to the fuzzy cortical representations, however, each stimulus will activate a somewhat different subset of assemblies. Therefore, the inhibition would be less pronounced. This mechanism does not preclude the filtering of redundant information as a model of sensory gating and altered M50 gating ratio, as suggested by Adler et al. (1982) and others.

\section{Conclusions}

The present results suggest that the interplay of processes evoked by the first stimulus and induced during the inter-stimulus interval influence the efficiency of the processing of the second of the two consecutive stimuli in a paired-stimulus design, and that smaller evoked and induced alpha and gamma activities contribute to the impaired processing of S2 and thus to the abnormal auditory gating ratio in schizophrenia patients. Relating the two indices, ERF and oscillatory activity, measured in the same design showed that the sources of the commonly used ERF/ERP measure of deficient sensory gating (i.e., the M50/P50 ratio) were confined to the STG and were related to broadly distributed sources of the evoked gamma band responses in the centroparietal regions (including posterior cingulated gyrus and precuneus) in healthy subjects but not in schizophrenia patients. Thus, the relationship of the two measures suggests that M50 generation is influenced by the activity that is not apparent when analyzing event-related potentials alone. In addition, the group difference in correlations suggests that the centro-parietal influence evident in the evoked gamma-band response is deficient in schizophrenia. The correlation of high M50 ratio and less induced alpha power decrease in broadly distributed fronto-cortical and centro-parietal brain regions in schizophrenia patients suggests that insufficient alpha desynchronization prior to S2 might contribute to abnormal M50 gating ratio. One might argue that the inherent differences between the two types of analyses might arise also as a function of different filter settings for ERF and time frequency analysis. Identical filter settings would yield identical data sets for unraveling the relationship between evoked fields and alpha band power, but identical filter settings could not be applied for recording and analysis of the gamma frequency up to $80 \mathrm{~Hz}$ - as determined in the present study. Thus, it is conceivable that the results for ERF/gating ratio and spectral power as a consequence were influenced by the fact that a low-pass filter was applied to the ERF analysis but not to the oscillatory activity analysis. A meta-analysis of the sensory gating studies in schizophrenia with special emphasis on methodological differences (Patterson et al., 2008) suggested that the choice of a high-pass filter indeed influences the gating ratio and that cut-off frequencies $>3 \mathrm{~Hz}$ might artificially increase P50 amplitude. The present data set does not allow specification whether and to what extent frequencies in the higher gamma range influenced the conventionally scored gating ratio. This would require opening of the low-pass filter settings in sensory gating designs in future ERP/ERF studies. Further work is needed to decide among several possible mechanisms of the impaired sensory gating in schizophrenia patients.

\section{Conflict of interest}

None of the authors has any actual or potential conflict of interest including any financial, personal or other relationships with other people or organizations that could inappropriately influence, or be perceived to influence, the presented work.

\section{Acknowledgments}

This work was supported by the German Research Foundation (Deutsche Forschungsgemeinschaft, DFG; Ro805/14). We thank Sarah Schulz, Petia Popova, and Ursula Lommen for their assistance in data collection and analysis and Dr. Patrick Berg and Dr. Christian Wienbruch for consultation on MEG methodology.

\section{References}

Ackenheil, M. Stotz-Ingenlath, G. Dietz-Bauer, R., Vossen, A. 1999, M.IN.I. Mini International Neuropsychiatric Interview, German Version 5.0.0 DSM IV. Psychiatrische Universitätsklinik München, München.

Adler, L.E., Pachtman, E., Franks, R.D., Pecevich, M., Waldo, M.C., Freedman, R., 1982. Neurophysiological evidence for a defect in neuronal mechanisms involved in sensory gating in schizophrenia. Biol. Psychiatry 17, 639-654.

Boutros, N.N., Brockhaus-Dumke, A., Gjini, K., Vedeniapin, A., Elfakhani, M., Burroughs, S Keshavan, M., 2009. Sensory-gating deficit of the N100 mid-latency auditory evoked potential in medicated schizophrenia patients. Schizophr. Res. 113, 339-346.

Bramon, E., Rabe-Hesketh, S., Sham, P., Murray, R.M., Frangou, S., 2004. Meta-analysis of the P300 and P50 waveforms in schizophrenia. Schizophr. Res. 70, 315-329.

Brenner, CA., Kieffaber, P.D., Clementz, B.A., Johannesen, J.K., Shekhar, A., O'Donnell, B.F. Hetrick. W.P., 2009. Event-related potential abnormalities in schizophrenia: a failure to "gate in" salient information? Schizophr. Res. 113, 332-338.

Brockhaus-Dumke, A., Mueller, R., Faigle, U., Klosterkoetter, J., 2008. Sensory gating revisited: relation between brain oscillations and auditory evoked potentials in schizophrenia. Schizophr. Res. 99, 238-249.

Clementz, B.A., Blumenf eld, L.D., 2001. Multichannel electroencephalographic assessment of auditory evoked response suppression in schizophrenia. Exp. Brain Res. 139, 377-390.

Clementz, B.A. Blumenf eld, L.D., Cobb, S., 1997. The gamma band response may account for poor P50 suppression in schizophrenia. NeuroReport 8, 3889-3893.

Debener, S., Herrmann, C.S., Kranczioch, C., Gembris, D., Engel, A.K., 2003. Top-down attentional processing enhances auditory evoked gamma band activity. NeuroReport $14,683-686$.

Edgar, J.C., Hanlon, F.M., Huang, M.X., Weisend, M.P., Thoma, R.J., Carpenter, B. Hoechstetter, K Canive, J.M. Miller, G.A. 2008. Superior temporal gyrus spectral abnormalities in schizophrenia. Psychophysiology 45, 812-824.

Freedman, R., Adler, L.E., Gerhardt, G.A., Waldo, M., Baker, N., Rose, G.M., Drebing, C. Nagamoto, H., Bickford-Wimer, P., Franks, R., 1987. Neurobiological studies of sensory gating in schizophrenia. Schizophr. Bull. 13, 669-678.

Gross, J., Kujala, J., Hamalainen, M., Timmermann, L., Schnitzler, A., Salmelin, R., 2001. Dynamic imaging of coherent sources: studying neural interactions in the human brain. Proc. Natl Acad. Sci. USA 98, 694-699.

Grunwald, T., Boutros, N.N., Pezer, N., von Oertzen, J., Fernandez, G., Schaller, C., Elger, C.E., 2003. Neuronal substrates of sensory gating within the human brain. Biol. Psychiatry 53, 511-519.

Haenschel, C., Bittner, R.A., Waltz, J., Haertling, F., Wibral, M., Singer, W., Linden, D.E. Rodriguez, E., 2009. Cortical oscillatory activity is critical for working memory as revealed by deficits in early-onset schizophrenia. J. Neurosci. 29, 9481-9489.

Hall, M.H., Taylor, G., Salisbury, D.F., Levy, D.L., 2010. Sensory gating event-related potentials and oscillations in schizophrenia patients and their unaffected relatives. Schizophr. Bull. 2010 Apr 2. [Epub ahead of print ], doi:10.1093/schbul/sbq027.

Hong, L.E., Summerfelt, A., McMahon, R.P., Thaker, G.K., Buchanan, R.W., 2004. Gamma/ betaoscillation and sensory gating deficit in schizophrenia. NeuroReport 15, 155-159.

Hong, L.E., Summerfelt, A., Mitchell, B.D., McMahon, R.P., Wonodi, I., Buchanan, R.W., Thaker, G.K., 2008. Sensory gating endophenotype based on its neural oscillatory pattern and heritability estimate. Arch. Gen. Psychiatry 65, 1008-1016.

Huang, M.X., Edgar, J.C., Thoma, R.J., Hanlon, F.M., Moses, S.N., Lee, R.R., Paulson, K.M., Weisend, M.P., Irwin, J.G., Bustillo, J.R., Adler, L.E., Miller, G.A., Canive, J.M., 2003. Predicting EEG responses using MEG sources in superior temporal gyrus reveals sourceasynchrony in patients with schizophrenia. Clin. Neurophysiol. 114, 835-850,

Jensen, O., Mazaheri, A., 2010. Shaping functional architecture by oscillatory alpha activity: gating by inhibition. Front. Hum. Neurosci. 4

Keil, J., Weisz, N., Paul-Jordanov, l., Wienbruch, C., 2010. Localization of the magnetic equivalent of the ERN and induced oscillatory brain activity. Neuroimage $51,404-411$. 
Kissler, J., Muller, M.M., Fehr, T., Rockstroh, B., Elbert, T., 2000. MEG gamma band activity in schizophrenia patients and healthy subjects in a mental arithmetic task and at rest. Clin. Neurophysiol. 111, 2079-2087.

Korzyukov, O., Pflieger, M.E. Wagner, M., Bowyer, S.M., Rosburg, T., Sundaresan, K. Elger, C.E., Boutros, N.N., 2007. Generators of the intracranial P50 response in auditory sensory gating. Neuroimage $35,814-826$.

Krause, C.M., 2006. Cognition- and memory-related ERD/ERS responses in the auditory stimulus modality. Prog. Brain Res. 159, 197-207.

Lecaignard, F., Bouet, R.M., Mattout, J., 2008. Comparing models of cortical anatomy for MEG source reconstruction. Biomag. (Abstract).

Leicht, G., Kirsch, V., Giegling, l., Karch, S., Hantschk, I., Moller, H.J., Pogarell, O., Hegerl, U., Rujescu, D., Mulert, C., 2010. Reduced early auditory evoked gamma-band response in patients with schizophrenia. Biol. Psychiatry 67, 224-231.

Lijffijt, M., Lane, S.D., Meier, S.L, Boutros, N.N., Burroughs, S., Steinberg, J.L., Gerard Moeller, F, Swann, A.C. 2009. P50, N100, and P200 sensory gating: relationships with behaviora inhibition, attention, and working memory. Psychophysiology 46, 1059-1068.

Maris, E., Oostenveld, R., 2007. Nonparametric statistical testing of EEG- and MEG-data. J. Neurosci. Meth. 164, 177-190.

Mayer, A.R., Hanlon, F.M., Franco, A.R., Teshiba, T.M., Thoma, R.J., Clark, V.P., Canive, J.M 2009. The neural networks underlying auditory sensory gating. Neuroimage 44 182-189.

Mulert, C., Leicht, G. Pogarell, O., Mergl, R., Karch, S., Juckel, G., Moller, H.J., Hegerl, U. 2007. Auditory cortex and anterior cingulate cortex sources of the early evoked gamma-band response: relationship to task difficulty and mental effort. Neuropsychologia 45, 2294-2306.

Nolte, G., 2003. The magnetic lead field theorem in the quasi-static approximation and its use for magnetoencephalography forward calculation in realistic volume conductors. Phys. Med. Biol. 48, 3637-3652.

Oldfield, R.C., 1971. The assessment and analysis of handedness: the Edinburgh inventory. Neuropsychologia 9.97-113.

Patterson, J.V., Hetrick, W.P., Boutros, N.N., Jin, Y., Sandman, C., Stern, H., Potkin, S. Bunney Jr., W.E., 2008. P50 sensory gating ratios in schizophrenics and controls: a review and data analysis. Psychiatry Res. 158, 226-247.

Popov, T., Jordanov, T., Rockstroh, B., Elbert, T., Merzenich, M.M., Miller, G.A., 2011. Specific cognitive training normalizes auditory sensory gating in schizophrenia: randomized trial. Biol. Psychiatry 69 (5), 465-471.
Potter, D., Summerfelt, A., Gold, J., Buchanan, R.W., 2006. Review of clinical correlates of P50 sensory gating abnormalities in patients with schizophrenia. Schizophr. Bull. $32,692-700$.

Pulvermuller, F., Keil, A., Elbert, T., 1999. High-frequency brain activity: perception or active memory? Trends Cogn. Sci. 3, 250-252.

Rosburg, T., Trautner, P., Fell, J., Moxon, K.A., Elger, C.E., Boutros, N.N., 2009 Sensory gating in intracranial recordings - the role of phase locking. Neuroimage 44, 1041-1049.

Tallon-Baudry, C., Bertrand, O., Delpuech, C., Permier, J., 1997. Oscillatory gamma-band $(30-70 \mathrm{~Hz})$ activity induced by a visual search task in humans.J. Neurosci. 17, 722-734.

Tallon-Baudry, C. Bertrand, O., Henaff, M.A., Isnard, J. Fischer, C., 2005. Attention modulates gamma-band oscillations differently in the human lateral occipital cortex and fusiform gyrus. Cereb. Cortex 15, 654-662.

Tallon-Baudry, C., Kreiter, A., Bertrand, O., 1999. Sustained and transient oscillatory responses in the gamma and beta bands in a visual short-term memory task in humans. Vis. Neurosci. 16, 449-459.

Thoma, R.J. Hanlon, F.M. Miller, G.A. Huang M. Weisend, M.P. Sanchez, F.P. Waldorf, V.A., Jones, A., Smith, A., Formoso, M.J., Canive, J.M., 2006. Neuropsychological and sensory gating deficits related to remote alcohol abuse history in schizophrenia. J. Int. Neuropsychol. Soc. 12, 34-44.

Thoma, R.J., Hanlon, F.M., Moses, S.N., Edgar, J.C., Huang, M., Weisend, M.P., Irwin, J., Sherwood, A., Paulson, K., Bustillo, J., Adler, LE., Miller, G.A., Canive, J.M., 2003. Lateralization of auditory sensory gating and neuropsychological dysfunction in schizophrenia. Am. J. Psychiatry 160, 1595-1605.

Weisser, R., Weisbrod, M., Roehrig, M., Rupp, A., Schroeder, J., Scherg, M., 2001. Is frontal lobe involved in the generation of auditory evoked P50? NeuroReport 12 , 3303-3307.

White, T.P., Joseph, V., O'Regan, E., Head, K.E., Francis, S.T., Liddle, P.F., 2010. Alphagamma interactions are disturbed in schizophrenia: a fusion of electroencephalography and functional magnetic resonance imaging. Clin. Neurophysiol. 121, 1427-1437.

Williams, T.J., Nuechterlein, K.H., Subotnik, K.L., Yee, C.M., 2011. Distinct neural generators of sensory gating in schizophrenia. Psychophysiology. 48, 470-478

Yee, C.M., Williams, T.J., White, P.M., Nuechterlein, K.H., Ames, D., Subotnik, K.L., 2010. Attentional modulation of theP50 suppression deficit in recent-onset and chronic schizophrenia. J. Abnorm. Psychol. 119, 31-39. 
Junghöfer, M., \& Peyk, P. (2004). Analysing electrical activity and magnetic fields in the brain. Matlab New's \& Notes, 2, 14-15.

Kahneman, D., \& Tversky, A. (1979). Prospect theory: An analysis of decision under risk. Econometrica, 47, 263-291.

Klein, G. A (1993). A recognition-primed decision (RPD) model of rapid decision. In: G. A. Klein, J. Orasanu, R. Calderwood, \& C. E. Zsambok (Eds.), Decision making in action: Models and methods (pp. 138-147). Norwood, NJ: Ablex.

Knutson, B., Fong, G. W., Bennett, S. M., Adams, C. M., \& Hommer, D. (2003). A region of mesial prefrontal cortex tracks monetarily rewarding outcomes: Characterization with rapid event-related fMRI. NeuroImage, 18, 263-272.

Knutson, B., Rick, S., Wimmer, G. E., Prelec, D., \& Loewenstein, G. (2007). Neural predictors of purchases. Neuron, 53, 147-156.

Koechlin, E., \& Summerfield, C. (2007). An information theoretical approach to prefrontal executive function. Trends in Cognitive Sciences, 11, 229-236.

Krain, A. L., Wilson, A. M., Arbuckle, R., Castellanos, F. X., \& Milham, M. P. (2006). Distinct neural mechanisms of risk and ambiguity: A meta-analysis of decision-making. NeuroImage, 32, 477-484.

Kringelbach, M. L. (2005). The human orbitof rontal cortex: Linking reward to hedonic experience. Nature Review's Neuroscience, 6 , 691-702.

Leng, Y., \& Zhou, X. (2010). Modulation of the brain activity in outcome evaluation by interpersonal relationship: An ERP study. Neuropsychologica, 48, 448-455.

March, J. G. (1978). Bounded rationality, ambiguity, and the engineering of choice. The Bell Journal of Economics, 9, 587-608.

Miller, G. A. (2010). Mistreating psychology in the decades of the brain. Perspectives on Psychological Science, 5, 716-743.

Miller, G. A., Elbert, T., Sutton, B. P., \& Heller, W. (2007). Innovative clinical assessment technologies: Challenges and opportunities in neuroimaging. Psychological Assessment, 19, 58-73.

Moser, J. S., \& Simons, R. F. (2009). The neural consequences of flipflopping: The feedback-related negativity and salience of reward prediction. Psychophysiology, 46, 313-320.

Neubert, F. X., \& Klein, M. C. (2010). What is driving inhibition-related activity in the frontal lobe? The Journal of Neuroscience, 30,4830 4832 .

Oldfield, R. C. (1971). The assessment and analysis of handedness: The Edinburgh Inventory. Neuropsychologica, 9, 97-113.

Peters, J., \& Büchel, C. (2009). Overlapping and distinct neural systems code for subjective value during intertemporal and risky decision making. Journal of Neuroscience, 29, 15727-15734.

Peyk, P., Schupp, H. T., Elbert, T., \& Junghoefer, M. (2008). Emotion processing in the visual brain: A MEG analysis. Brain Topography, 20, 205-215.
Rangel, A., Camerer, C., \& Montague, P. R. (2008). A framework for studying the neurobiology of value-based decision making. Nature Reviews Neuroscience, 9, 545-556.

Rushworth, M. F., \& Behrens, T. E. (2008). Choice, uncertainty and value in prefrontal and cingulated cortex. Nature Neuroscience, 11 , 389-397.

Sanfey, A. G., Hastie, R., Colvin, M. K., \& Grafman, J. (2003). Phineas gauged: Decision-making and the human prefrontal cortex. Neurops ychologia, 4l, 1218-1229.

Sanfey, A. G., Loewenstein, G., McClure, S. M., \& Cohen, J. D. (2006). Neuroeconomics: Cross-currents in research on decision-making. Trends in Cognitive Sciences, 10, 108-116.

Sanfey, A. G., \& Chang, L. J. (2008). Multiple systems in decision making. Annals of the New York Academy of Sciences, 1128, 53-62

Sasaki, K., \& Gemba, H. (1986). Electrical activity in the prefrontal cortex specific to no-go reaction of conditioned hand movement with color discrimination in the monkey. Experimental Brain Research, 64, 603-606.

Savage, L. J. (1954). The foundations of statistics. New York: Wiley.

Schmitt, B. M., Schlitz, K., Zaake, W., Kutas, M., \& Münte, T. F. (2001). An electrophysiological analysis of the time course of conceptual and syntactical encoding during tacit picture naming. Journal of Cognitive Neuroscience, 13, 510-522.

Schultz, W. (2004). Neural coding of basic reward terms of animal learning theory, game theory, microeconomics and behavioural ecology. Current Opinion in Neurobiology, 14, 139-147.

Smith, B. W., Mitchell, D. G., Hardin, M. G., Jazbec, S., Fridberg, D., Blair, R. J., \& Ernst, M. (2009). Neural substrates of reward magnitude, probability, and risk during a wheel of fortune decision-making task. NeuroImage, 44, 600-609.

Steffen, A., Rockstroh, B., \& Jansma, B. (2009). Brain evoked potentials reflect how other people's emotions influence decisions. Journal of Neuroscience, Psychology, and Economics, 2, 32-40.

Tversky, A., \& Kahneman, D. (1981). The framing of decisions and the psychology of choice. Science, $211,453-458$

von Neumann, J., \& Morgenstern, O. (1947). Theory of games and economic behavior. Princeton: Princeton University Press.

Weisend, M. P., Hanlon, F. M., Huang, M. X., Thoma, R. J., Edgar, J. C., Lee, R. R., \& Canive, J. M. (Under review). Detecting and characterizing hippocampal activity with magnetoencephalography.

Yu, R., \& Zhou, X. (2009). To bet or not to bet? The error negativity or error-related negativity associated with risk-taking choices. Journal of Cognitive Neuroscience, 21, 684-696 